\title{
Differential Constraints Compatible with Linearized Equations
}

\author{
Ahmet SATIR \\ Middle East Technical University, Department of Physics, \\ 06531 Ankara, Turkey \\ Correspondence address: \\ Koza Sokak 136/1, G.O.P. 06670 Ankara, Turkey \\ Fax: +90 312437 8634 \\ Received January 15, 1998; Revised July 30, 1998; Accepted July 31, 1998
}

\begin{abstract}
Differential constraints compatible with the linearized equations of partial differential equations are examined. Recursion operators are obtained by integrating the differential constraints.
\end{abstract}

One of the standard ways for determining particular solutions to partial differential equations is to reduce them to ordinary differential equations which are easier to solve. The classical work of Lie about group-invariant solutions generalizes well-known methods for finding similarity solutions and other basic reduction methods [1]. Bluman and Cole [2] proposed a generalization of Lie's method for finding group-invariant solutions, which they named the "nonclassical" method. In this approach, one replaces the condition for the invariance of the given system of differential equations by the weaker condition for the invariance of the combined system consisting of the original differential equations along with the equations requiring the group invariance of the solutions. P.J. Olver and P. Rosenau proposed a generalization of the nonclassical method [3, 41. They showed that many known reduction methods, including the classical and nonclassical methods, partial invariance, and separation of variables can be placed into a general framework. In their formulation, the original system of partial differential equations can be enlarged by appending additional differential constraints (side conditions), such that the resulting overdetermined system of partial differential equations satisfy compatibility conditions.

This work discusses differential constraints compatible with the linearized equations of partial differential equations instead of the partial differential equations themselves. The relation between differential constraints and recursion operators are examined. For the type of equations in the form $q_{t}=P\left(x, t, q, q_{x}, q_{x x}\right)$ and $q_{t}=P\left(q, q_{x}, q_{x x}, q_{x x x}\right)$, recursion operators are obtained by integrating the compatible differential constraints. A new type 
of integrable equations, which are generalizations of the integrable equations of Fokas and Svinolupov, are given. Results are also compared with Fokas' generalized symmetry [5] and Mikhailov-Shabat-Sokolov's formal symmetry approaches [6, 7].

We can describe the differential constraint method for evolutionary equations [9]

$$
q_{t}=P\left(x, t, q, q_{x}, q_{x x}, \ldots\right)
$$

in the following way. First we linearize the given differential equation. In other words, we replace $q$ (and its derivatives) in (11) by $q+\epsilon \Psi$ and differentiate both sides of the resulting expression with respect to $\epsilon$ and take the limit $\epsilon \rightarrow 0$, i.e.,

$$
\Psi_{t}=D_{P}(\Psi)
$$

where $D_{P}$ is the Fréchet derivative [1]. The equation above can also be written as

$$
\Psi_{t}=\sum_{i=0}^{N} P_{i} \Psi_{i}=\sum_{i=0}^{N} \frac{\partial P}{\partial q_{i}} \Psi_{i}
$$

where $N$ is the order of differential equation, $q_{0}=q, q_{1}=q_{x}, q_{2}=q_{x x}, \Psi_{0}=\Psi, \Psi_{1}=\Psi_{x}$, $\Psi_{2}=\Psi_{x x}$, and so on. In the classical symmetry approach, (2) is the main equation, where $\Psi$ is the symmetry of the differential equation, which is a function of $x, t, q_{i}$.

The compatible differential constraint is

$$
H \Psi=0,
$$

where $H$ depends on $x, t, q_{i}$. If its order (highest derivative in $H$ ) is $N$, then (团) may be written as

$$
\Psi_{N}=\sum_{i=0}^{(N-1)} A_{i} \Psi_{i}
$$

where $A_{0}, A_{1}, \ldots, A_{N-1}$ are functions of $x, t, q_{i}$. Compatibility of (5) and (2) is given by

$$
\Psi_{N, t}-\Psi_{t, N}=0
$$

Using (2) and (5), rewlation (6) leads to

$$
\sum_{i=0}^{N-1} \Psi_{i} W_{i}=0 .
$$

Letting

$$
W_{i}=0,
$$

we obtain a system of partial differential equations among $P_{i}, A_{i}$ and their partial derivatives. The solution of this system will determine the differential constraint (画, which can be integrated to give

$$
\Phi \Psi=0,
$$

where $\Phi$ is the recursion operator. 
Let us now consider differential equations of the following form

$$
q_{t}=f\left(x, t, q, q_{x}, q_{x x}\right) .
$$

The linearized form of (10) can be given as

$$
\Psi_{t}=\gamma \Psi_{x x}+\alpha \Psi_{x}+\beta \Psi
$$

where $\alpha, \beta, \gamma$ are functions of $x, t, q, q_{x}, q_{x x}$. We assume a differential constraint having the same order as (10) in the form

$$
\Psi_{x x}=A \Psi_{x}+B \Psi,
$$

where $A$ and $B$ are functions of $x, t, q, q_{x}, q_{x x}$. The compatibility condition between (11) and (12) will give the following evolution equations

$$
\begin{aligned}
A_{t}= & \alpha_{x x}+\alpha_{x} A+\gamma_{x x} A+2 \gamma_{x} A_{x}+\gamma_{x} A^{2}+2 \gamma_{x} B \\
& +2 \beta_{x}+A_{x x} \gamma+A_{x} \alpha+2 A_{x} \gamma A+2 B_{x} \gamma, \\
B_{t}= & 2 \alpha_{x} B+\gamma_{x x} B+2 \gamma_{x} B_{x}+\gamma_{x} A B+\beta_{x x} \\
& -\beta_{x} A+2 A_{x} \gamma B+B_{x x} \gamma+B_{x} \alpha .
\end{aligned}
$$

The solutions of the system (13)-(14) can be given with the linearized equation

$$
\Psi_{t}=\eta \Psi_{x x}+\left[\frac{2 \eta r_{q q}}{r_{q}} q_{x}+2 \eta r+\eta_{1}\right] \Psi_{x}+\left[\frac{\eta\left(r_{q q q} r_{q}-r_{q q}^{2}\right)}{r_{q}^{2}} q_{x}^{2}+2 \eta r_{q} q_{x}\right] \Psi,
$$

and compatible differential constraint

$$
\Psi_{x x}=\left[\frac{q_{x x}}{q_{x}}-\frac{q_{x} r_{q q}}{r_{q}}-r\right] \Psi_{x}+\left[\frac{r q_{x x}}{q_{x}}-\frac{\left(r_{q q q} r_{q}-r_{q q}^{2}\right) q_{x}^{2}}{r_{q}^{2}}-2 r_{q} q_{x}-r_{x}\right] \Psi .
$$

Here $\eta, \eta_{1}$ are function of $x, t$, and $r$ is a function of $x, t, q$, such that they satisfy

$$
\begin{aligned}
& 2 r_{x} \eta+\eta_{x} r=0, \quad 2 r_{t} \eta+\eta_{t} r=0 \\
& r_{t}-\frac{r}{4 \eta}\left(-2 \eta \eta_{x x}+\eta_{x}^{2}-2 \eta_{1} \eta_{x}+4 \eta \eta_{1, x}\right)=0 .
\end{aligned}
$$

It is interesting to note that in this classification the explicit time dependence is crucial for obtaining equations (17)-(18), which do not have any derivative with respect to $q$, although $r$ depend explicitly on $q$. The differential constraint (16) can be integrated to give

$$
\Phi=D+\frac{r_{q q} q_{x}}{r_{q}}+r+q_{x} D^{-1} r_{q}+q_{x} D^{-1}\left(\frac{r_{q q x} r_{q}-r_{q q} r_{q x}}{r_{q}^{2}}\right)
$$

where $\left(D^{-1} f\right)(x)=\int_{-\infty}^{x} f(\zeta) d \zeta$. Because of the last term in (19) and the explicit dependence of $x$ and $t$ on $r, \Phi$, as given by (19), is new recursion operator which is a 
generalization of the recursion operators obtained by Fokas [5] and Ibragimov-Shabat [10]. The new integrable equation takes the following form

$$
q_{t}=\eta q_{x x}+\frac{\eta r_{q q} q_{x}^{2}}{r_{q}}+\left(2 \eta r+\eta_{1}\right) q_{x}
$$

Although (20) looks like the integrable equations classified by Fokas [5], they are different, because of the explicit $x, t$ dependence of $r, \eta$ and $\eta_{1}$, which are subject to conditions (17) $-(18)$. Using transformation $q(x, t) \rightarrow r(q(x, t), x, t)$ [5] one obtains

$$
r_{t}=\eta r_{x x}+\left(2 \eta r+\eta_{1}\right) r_{x}-\eta\left(r_{q, x} q_{x}+r_{, x x}\right)-\left(2 \eta r+\eta_{1}\right) r_{, x}+r_{, t},
$$

where $r_{, x}, r_{, t}$ denotes partial derivatives with respect to $x, t$. However, in the limit $r(q, x, t) \rightarrow r(q)$, we recover the equations classified by Fokas [5, Ibragimov-Shabat 10] and Olver [8]. Moreover, equations (21) are more general than the once obtained by Svinolupov [6], since he analyzed the case $q_{t}=F\left(x, q, q_{x}, q_{x x}\right)$, in which there is no explicit time dependence. It is interesting to note that in the limit $r(q, x, t) \rightarrow r(q, t)$ (using the conditions (17) $-(18)$ ), equation (20) will give Svinolupov's equation [6]

$$
q_{t}=\eta^{\prime} q_{x x}+\frac{\eta^{\prime} r_{q q} q_{x}^{2}}{r_{q}}+\left(2 \eta^{\prime} r-\frac{\eta_{t}^{\prime}}{2 \eta^{\prime}} x+\epsilon\right) q_{x}
$$

where $\eta^{\prime}$ and $\epsilon$ are functions of $t$, and $r$ is a function of $q$ and $t$.

Equations (17) can be integrated to give $\eta=\frac{a}{r^{2}}$, where $a=a(q)$. Substitution of $\eta$ in (18) will give

$$
a r_{x x} r-2 a r_{x}^{2}+r_{x} \eta_{1} r^{3}+\eta_{1, x} r^{4}-r_{t} r^{3}=0 .
$$

One particular solution can be given by solving the last two terms in the above equation. This case $(r(q, t))$ corresponds to equation (22). Equation (23) is a coupled second order partial differential equation and the general solution can be given by the method of characteristics [11]. It is hoped that the general solution will appear in a future work.

Next, we consider differential equations of the following general form, which includes the KdV equation,

$$
q_{t}=P\left(q, q_{x}, q_{x x}, q_{x x x}\right) .
$$

The linearization of (24) takes the form

$$
\Psi_{t}=\alpha \Psi_{x x x}+\beta \Psi_{x x}+\gamma \Psi_{x}+\delta \Psi,
$$

where $\alpha, \beta, \gamma, \delta$ are functions of $q, q_{x}, q_{x x}, q_{x x x}$. We consider the differential constraint having the same order as (24), i.e.,

$$
\Psi_{x x x}=A \Psi_{x x}+B \Psi_{x}+C \Psi
$$

where $A, B$ and $C$ are functions of $q, q_{x}, q_{x x}, q_{x x x}$. The compatibility will determine algebraic equations

$$
B=-\frac{2 \alpha}{3 \eta}, \quad C=-\frac{1}{3 \eta}\left(\alpha_{x}-2 \alpha A+2 \beta\right)
$$


and evolution equations

$$
\begin{aligned}
A_{t}= & \alpha_{x} A+\beta_{x}+A_{x x x} \eta+3 A_{x x} \eta A+3 A_{x}^{2} \eta+A_{x} \alpha+3 A_{x} \eta A^{2}, \\
\alpha_{t}= & \frac{1}{2}\left(2 \alpha_{x x x} \eta-3 \alpha_{x x} \eta A-3 \alpha_{x} A_{x} \eta+2 \alpha_{x} \alpha\right. \\
& \left.-3 \beta_{x x} \eta+6 \beta_{x} \eta A+6 A_{x} \beta \eta+3 \varepsilon_{t} \eta\right) \\
\beta_{t}= & \frac{1}{4}\left(3 \alpha_{x x x} \eta A-6 \alpha_{x x} \eta A^{2}-3 \alpha_{x} A_{x x} \eta-12 \alpha_{x} A_{x} \eta A+4 \alpha_{x} \beta\right. \\
& \left.+\beta_{x x x} \eta-6 \beta_{x x} \eta A+4 \beta_{x} \alpha+12 \beta_{x} \eta A^{2}+6 A_{x x} \beta \eta+24 A_{x} \beta \eta A\right),
\end{aligned}
$$

where $\eta$ is constant and $\epsilon$ is a function of $t$.

The linearized form of the first class is

$$
\Psi_{t}=\eta \Psi x x x+\left[\frac{\rho_{1}}{2} q_{x}^{2}+\rho\right] \Psi_{x}+\rho_{q} q_{x} \Psi,
$$

with compatible differential constraint

$$
\Psi_{x x x}=\frac{q_{x x}}{q_{x}} \Psi_{x x}-\left[\frac{\rho_{1}}{3 \eta} q_{x}^{2}+\frac{2 \rho}{3 \eta}\right] \Psi_{x}-\frac{-2 \rho q_{x x}+3 \rho_{q} q_{x}^{2}}{3 \eta q_{x}} \Psi,
$$

where $\rho_{1}$ is constant and $\rho$ is a function of $q$ with the condition

$$
\rho_{q q q}+\frac{4 \rho_{1}}{3 \eta} \rho_{q}=0
$$

The recursion operator can be obtained by integrating (32). This leads to

$$
\Phi=D^{2}+\frac{2 \rho}{3 \eta}+\frac{\rho_{1}}{3 \eta} q_{x}^{2}-\frac{\rho_{1}}{3 \eta} q_{x} D^{-1} q_{x x}+\frac{q_{x}}{\eta} D^{-1} \rho_{q} .
$$

The integrable equation is in the form

$$
q_{t}=\eta q_{x x x}+\frac{\rho_{1}}{6} q_{x}^{3}+\rho q_{x}
$$

The second class is given by the linearized equation

$$
\Psi_{t}=\eta \Psi_{x x x}+\left[\epsilon_{1} q_{x}^{2}+2 \epsilon_{1} \epsilon_{2} q_{x}+2 \epsilon_{3}\right] \Psi
$$

with compatible differential constraint

$$
\Psi_{x x x}=\frac{q_{x x}}{q_{x}+\epsilon_{2}} \Psi_{x x}-\left[\frac{\epsilon_{1}}{3 \eta} q_{x}^{2}+\frac{2 \epsilon_{1} \epsilon_{2}}{3 \eta} q_{x}+\frac{2 \epsilon_{3}}{3 \eta}\right] \Psi_{x}-\frac{\left(\epsilon_{2}^{2} \epsilon_{1}-2 \epsilon_{3}\right) q_{x x}}{3 \eta\left(q_{x}+\epsilon_{2}\right)} \Psi,
$$

where $\epsilon_{1}, \epsilon_{2}, \epsilon_{3}$ are constants. The recursion operator can be obtained by integrating (37), which gives

$$
\Phi=D^{2}+\frac{2 \epsilon_{3}}{3 \eta}+\frac{\epsilon_{2}}{3 \eta} q_{x}^{2}+\frac{2 \epsilon_{1} \epsilon_{2}}{3 \eta} q_{x}-\frac{\epsilon_{1}}{3 \eta}\left(q_{x}+\epsilon_{2}\right) D^{-1} q_{x x} .
$$

The integrable equation is in the form

$$
q_{t}=\eta q_{x x x}+\frac{\epsilon_{1}}{6} q_{x}^{3}+\frac{\epsilon_{1} \epsilon_{2}}{2} q_{x}^{2}+\epsilon_{3} q_{x} .
$$


Equations (35), (39) were classified by Fokas [5], Ibragimov-Shabat [10]. We obtained the recursion operators (34), (38) by integrating the differential constraints.

The third type of equation is given by the linearized equation

$$
\Psi_{t}=\lambda_{5} \Psi_{x x x}+\frac{3}{2} \lambda_{3} \lambda_{5} \Psi_{x x}+\frac{3}{2} \lambda_{4} \lambda_{5} \Psi_{x}+\lambda_{6} \Psi
$$

and differential constraint

$$
\begin{aligned}
\Psi_{x x x} & =\left[\frac{q_{x x x}+\lambda_{1} q_{x x}+\lambda_{2} q_{x}}{q_{x x}+\lambda_{1} q_{x}+\lambda_{2} q}-\lambda_{3}\right] \Psi_{x x} \\
& +\left[\frac{\left.\lambda_{3(} q_{x x x}+\lambda_{1} q_{x x}+\lambda_{2} q_{x}\right)}{q_{x x}+\lambda_{1} q_{x}+\lambda_{2} q}-\lambda_{4}\right] \Psi_{x}+\frac{\lambda_{4}\left(q_{x x x}+\lambda_{1} q_{x x}+\lambda_{2} q_{x}\right)}{q_{x x}+\lambda_{1} q_{x}+\lambda_{2} q} \Psi .
\end{aligned}
$$

The recursion operator is

$$
\Phi=D^{2}+\lambda_{3} D+\lambda_{4}
$$

The integrable equation is given as

$$
q_{t}=\lambda_{5} q_{x x x}+\frac{3}{2} \lambda_{3} \lambda_{5} q_{x x}+\frac{3}{2} \lambda_{4} \lambda_{5} q_{x}+\lambda_{6} q
$$

where $\lambda_{1}, \lambda_{2}, \lambda_{3}, \lambda_{4}, \lambda_{5}$ and $\lambda_{6}$ are constants. Note that Rabelo and Tanenblat also obtained linear equation using the classification method of pseudo-spherical surfaces with Gaussian curvature (-1) [12].

According to Fokas, an integrable equation has infinitely many generalized symmetries which are the solutions of (2). The existence of generalized symmetry manifest itself by the existence of an admissible Lie-Bäcklund operator. The existence of infinitely many symmetries is expressed by the existence of a recursion operator. There is a close relationship between Lie-Bäcklund operator and linearized equation [5]. Because, if Fokas' admissible Lie-Bäcklund operator is applied on the evolution equation, we obtain the Fréchet derivative of our differential equation under consideration or linearized form of our differential equation. Recursion operators, in our method, are obtained by the integration of the compatible differential constraints.

Let us briefly recall Olver-Fokas symmetry approach and the differential constraint test:

Olver-Fokas symmetry test: The equation $q_{t}=P[q]$ is integrable if there exists infinitely many non-Lie point symmetries, or equivalently, one non-Lie point symmetry and a recursion operator. The recursion operator and the time-independent part of the linearized differential equation form a Lax pair $\Re_{t}+\left[\Re, D_{P}\right]=0$.

Differential constraint test: The equation $q_{t}=P[q]$ is integrable if there exists a differential constraint $H \Psi=0$, such that it is compatible with the linearized equation $\Psi_{t}=D_{P}(\Psi)$. The compatibility condition is $H_{t}+\left[H, D_{P}\right]=0$.

There are several methods to examine the integrability of nonlinear partial differential equations [13], although in two dimensions most of these methods imply each other. Infinite sets of conservation laws, infinite number of symmetries, and the bi-Hamiltonian 
structure, are a number of remarkable properties, to name but three. The recursion operator plays an important role in the formulation of these recursive properties. Firstly, the family of integrable equations can be written in a compact form using recursion operators. The other role of recursion operator is associated with the Hamiltonian treatment of integrable equations. Recursion operators determine Hamiltonian structures through certain Poisson brackets [14]. Further analysis of the recursion operators leads to the concept of bi-Hamiltonian structures, which can be given by factorizing the recursion operator.

The main idea in this work is to give a new definition of integrability. A partial differential equation is integrable if its linearized equation is compatible with a differential constraint. Using differential constraints compatible with the linearized equations, we also give an answer to the question "The deep connection between the direct reduction and recursion operators" of Olver [\$].

\section{Acknowledgments}

I would like to thank Norbert Euler and the referee for their constructive comments.

\section{References}

[1] Olver P.J., Applications of Lie Groups to Differential Equations, Springer-Verlag, Berlin, 1993.

[2] Bluman G.W. and Cole J.D., The General Similarity Solutions of the Heat Equation, J. Math. Mech., 1969, V.18, 1025-1042.

[3] Olver P.J. and Rosenau P., Group Invariant Solutions of Differential Equations, SAIM J. Appl. Math., 1987, V.47, 263-278.

[4] Olver P.J. and Rosenau P., The Construction of Special Solutions to Partial Differential Equations, Phys. Lett. A, 1986, V.11, 107-112.

[5] Fokas A.J., A Symmetry Approach to Exactly Solvable Evolution Equations, J. Math. Phys., 1980, V.21, 1318-1325.

[6] Svinolupov S.I., 1985 Second-Order Evolution Equations with Symmetries, Russian Math. Surveys, 1980, V.40, 241-242.

[7] Mikhailov A.V., Shabat A.B. and Sokolov V.V., in: What is Integrability, ed. V.E. Zakharov, Springer-Verlag, Berlin, 1991.

[8] Olver P.J., Direct Reduction and Differential Constraints, Proc.R.Soc.Lon. A, 1994, V.444, 509-523.

[9] Satır A., Differential Constraints, Recursion Operators and Logical Integrability, Inter. J. Theor. Phys., 1997, V.10, 2099-2105.

[10] Ibragimov N.Kh. and Shabat A.B., Evolutionary Equations with Nontrivial Lie-Bäcklund Group, Funcl. Anal. Appl., 1980, V.14, 19-28.

[11] Sneddon I., Elements of Partial Differential Equations, McGraw-Hill Kogakuska Ltd., 1957.

[12] Rabelo M.L. and Tanenblat K., A Classification of Pseudospherical Surface Equations of Type $u_{t}=$ $u_{x x x}+G\left(u, u_{x}, u_{x x}\right)$, J. Math. Phys., 1992, V.33, 537-549.

[13] Fokas A.S., Symmetry and Integrability, Stud. Appl. Math., 1987, V.77, 253-299.

[14] Fordy A.P. and Gibbons J., Factorization of Operators I. Miura Transformations, J. Math. Phys., 1980, V.21, 2508-2510. 